\title{
La realidad poliédrica de la adherencia al tratamiento analgésico
} The multifaceted reality of adherence to analgesic treatment

La no adherencia a los tratamientos médicos es muy común y constituye un reto para el profesional sanitario, al incidir en la disminución de la eficacia de los tratamientos prescritos. De hecho, al considerar el tratamiento farmacológico de patologías crónicas, las tasas promedio de incumplimiento terapéutico son elevadas, del 20,6 \% según algunos estudios [1], o incluso del $50 \%$ según otros [2]. En el caso concreto del dolor crónico, el porcentaje promedio de incumplimiento de las prescripciones es del $40 \%$ [3]. El 29,9\% de los pacientes con dolor crónico no oncológico toma menos medicación de la prescrita y el 13,7\% más [4]. Con esa tasa de incumplimiento, la elección del tratamiento farmacológico no solo debería basarse en el diagnóstico del dolor, sino que también debería tener en consideración los riesgos de no adherencia terapéutica [3].

La falta de adherencia terapéutica es un constructo poliédrico que puede ser contemplado desde diferentes perspectivas, en el que pueden confluir múltiples causas y que, además, pueden incidir de manera conjunta. Bosworth y cols. (2018) (5), en un intento de sistematizar las variables asociadas con la adherencia a la medicación, describen diferentes barreras que pueden condicionar el adecuado cumplimiento terapéutico. En concreto, consideran barreras relacionadas con el propio paciente (olvido, autocontrol de los síntomas, interrupciones del tratamiento por causas diversas, mala comprensión de las instrucciones, inadecuado conocimiento sobre la patología y los medicamentos utilizados para tratarla, determinadas creencias culturales o religiosas sobre la salud y la medicación, falta de apoyo social, estilo de vida caótico o depresión y otras condiciones de salud mental), barreras relacionadas con el propio medicamento (número de pastillas, frecuencia de dosificación, efectos secundarios o efectos adversos del medicamento, tomar varios medicamentos al mismo tiempo, duración de la terapia, tiempo de espera de la farmacia, tamaño y sabor de la pastilla o instrucciones de dosificación), barreras relacionadas con el propio prescriptor (falta de confianza en el médico, mala comunicación con el mismo, presión asistencial, creencias culturales, de salud y/o religiosas que pueden generar disparidad entre el médico y el paciente), y también barreras relacionadas con el propio sistema de salud (falta de cobertura de ciertos tratamientos, dificultad para concertar citas, o barreras para acceder a recetas iniciales o reabastecimientos] [5]. Así pues, las posibles causas de la inadecuada adherencia terapéutica son múltiples y procedentes de distintos ámbitos.

Una de las barreras descritas más arriba está relacionada con las características del propio paciente y, más concretamente, con aspectos vinculados a la salud mental. En este número de la Revista de la Sociedad Española del Dolor se publica un interesante trabajo que revisa la incidencia de los factores psicológicos (del propio paciente) en la no adherencia terapéutica. Concretamente, el trabajo titulado "Factores psicológicos asociados a la adherencia al tratamiento analgésico en pacientes con dolor crónico: revisión sistemática de la literatura", realizado por Palomo y cols., se ha centrado en revisar sistemáticamente aquellas publicaciones que hubiesen estudiado la relación entre variables psicológicas y la falta de seguimiento de las recomendaciones o prescripción realizadas por el médico, excluyendo expresamente un tema importante, como es el de la interacción paciente-facultativo y de la interacción del paciente con sus familiares o redes de apoyo. De los 1184 artículos potenciales, y tras un riguroso proceso de elegibilidad mediante procedimientos estandarizados, fueron seleccionados 14, que incluyeron tanto trabajos transversales como longitudinales. Las variables psicológicas que se relacionaron con la adherencia terapéutica fueron: depresión, ansiedad, afecto negativo, estrategias de afrontamiento y catastrofismo. En la discusión los autores remarcan la complejidad del tema y la diversidad de variables, personales o contextuales, que no han sido objeto de este análisis. Lejos de ser una limitación que reste importancia a la revisión, posiblemente sea una virtud que haya permitido 
centrar el foco del estudio en aspectos intraindividuales. Los resultados de la revisión remarcaron la relativa consistencia de la depresión y de la ansiedad, o de la conjunción de ambas, en la falta de adherencia al tratamiento. También remarcaron la necesidad de más estudios que contemplasen otras variables psicológicas que pudieran ser potencialmente significativas en la adherencia al tratamiento. Una revisión interesante que merece ser leída con detenimiento.

Sin embargo, no puedo resistirme a abrir un poco más el foco, comentando algunos aspectos relacionados con la psicología que van más allá de esta revisión, y que son importantes áreas de investigación sobre variables predictoras de la no adherencia terapéutica. Existen evidencias de que las preocupaciones y creencias del paciente predicen la falta de adherencia a la medicación, y no solo eso, sino además la dirección de ese incumplimiento. El uso excesivo estaría relacionado con mayor percepción de gravedad de la enfermedad, mayor necesidad percibida de medicación y menor preocupación sobre la adicción, mientras que la infrautilización estaría relacionada con mayor preocupación sobre los efectos secundarios y menor confianza en el médico que prescribe [6-9]. Por tanto, la calidad de la relación médico-paciente es relevante, dado que la satisfacción del paciente ha demostrado ser primordial para minimizar alguna de las barreras que favorecen la no adherencia terapéutica (8), existiendo propuestas que buscan mejorar esa relación desde marcos teóricos contrastados $[5,6,10]$.

Si bien las intervenciones para mejorar la inadecuada adherencia terapéutica no intencionada pueden realizarse mediante procedimientos relativamente sencillos y generalizables (recordatorios, simplificación de las dosis, educación, etc.), la no adherencia intencional es más compleja y va a requerir de intervenciones adaptadas a las características de cada paciente, en las que la valoración de los factores psicológicos asociados a la adherencia al tratamiento analgésico deberá ser considerada.

A. Castel Riu Unidad de Dolor. Hospital Universitari Joan XXIII. Tarragona. Grup Multidisciplinari d'Investigació en Dolor. Institut d'Investigació Sanitària Pere Virgili. Tarragona, España

Correspondencia: Antoni Castel Riu antonicastel.hj23.ics@gencat.cat

\section{BIBLIOGRAFÍA}

1. DiMateo MR, Giordani PJ, Lepper HS, Croghan TW. Patient adherence and medical treatment outcomes: a meta-analysis. Med Care. 2002;40(9):794-811. DOl: 10.1097/00005650-200209000-00009.

2. Zullig LI, Peterson ED, Bosworth HB. Ingredients of successful interventions to improve medication adherence. JAMA. 2013;24(24):2611-2.

3. Timmerman L, Stronks DL, Groeneweg JG, Huygen FJ. Prevalence and determinants of medication nonadherence in chronic pain patients: a systematic review. Acta Anaesthesiol Scand. 2016;60(4):416-31. DOI: $10.1111 /$ aas. 12697.

4. Broekmans S, Dobbels F, Milisen K, Morlion B, Vanderschueren S. Medication adherence in patients with chronic non-malignant pain: is there a problem? Eur J Pain. 2009;13(2):115-23. DOI: 10.1016/j. ejpain.2008.02.010.

5. Bosworth HB, Blalock DV, Hoyle RH, Czajkowski SM, Voils Cl. The role of psychological science in efforts to improve cardiovascular medication adherence. Am Psychol. 2018;73(8):968-80. D0I: 10.1037/ amp0000316.

6. Matthias MS, Krebs EE, BergmanAA, Coffing JM, Bair MJ. Communicating about opioids for chronic pain: A qualitative study of patient attributions and the influence of the patient-physician relationship. Eur J Pain. 2014;18(6):835-43. DOI: 10.1002/j.1532-2149.2013.00426.x.

7. Nicklas LB, Dunbar M, Wild M. Adherence to pharmacological treatment of non-malignant chronic pain: The role of illness perceptions and medication beliefs. Psychol Health. 2010;25(5):601-15. D0I: 10.1080/08870440902783610.

8. Chou PL, Rau KM, Yu TW, Huang TL, Sun JL, Wang SY, et al. Patient-clinician relationship seems to affect adherence to analgesic use in cancer patients: a cross sectional study in a Taiwanese population. Int J Qual Health Care. 2017;29(7):935-40. D0I: 10.1093/intqhc/mzx134.

9. Timmerman L, Stronks DL, Huygen FJ. The Relation Between Patients' Beliefs About Pain Medication, Medication adherence, and treatment outcome in chronic pain patients: A prospective study. Clin J Pain. 2019;35(12):941-7. DOI: 10.1097/AJP.0000000000000760.

10. Timmerman L, Stronks DL, Huygen FJ. The design of a theory-based intervention to improve medication adherence in chronic pain patients. Curr Med Res Opin. 2017;33(7):1293-01. DOI: 10.1080/03007995.2017.1314955. 